University of South Carolina

Scholar Commons

$1-15-2001$

\title{
Semiclassical Application of the Mocciller Operators in Reactive Scattering
}

Sophya Garashchuk

University of South Carolina--Columbia, sgarashc@chem.sc.edu

John C. Light

Follow this and additional works at: https://scholarcommons.sc.edu/chem_facpub

Part of the Chemistry Commons

\section{Publication Info}

Published in Journal of Chemical Physics, Volume 114, Issue 3, 2001, pages 1060-1064.

http://jcp.aip.org/

(C) 2001 by American Institute of Physics

This Article is brought to you by the Chemistry and Biochemistry, Department of at Scholar Commons. It has been accepted for inclusion in Faculty Publications by an authorized administrator of Scholar Commons. For more information, please contact digres@mailbox.sc.edu. 


\title{
Semiclassical application of the Møller operators in reactive scattering
}

\author{
Sophya Garashchuk and John C. Light \\ James Franck Institute, University of Chicago, Chicago, Illinois 60637
}

(Received 19 July 2000; accepted 25 October 2000)

\begin{abstract}
Møller operators in the formulation of reaction probabilities in terms of wave packet correlation functions allow us to define the wave packets in the interaction region rather than in the asymptotic region of the potential surface. We combine M $\phi$ ller operators with the semiclassical propagator of Herman and Kluk. This does not involve further approximations and can be used with any initial value representation (IVR) semiclassical propagator. Time propagation in asymptotic regions of the potential due to Mbller operators reduces the oscillations of the propagator integrand and improves convergence of the results with respect to the number of trajectories. The effectiveness of Møller operators for semiclassical reaction probability calculation is demonstrated for the collinear hydrogen exchange reaction. Full convergence is achieved and the number of classical trajectories is reduced by a factor of 10 compared to the calculation without Mфller operators. (c) 2001 American Institute of Physics. [DOI: 10.1063/1.1333408]
\end{abstract}

\section{INTRODUCTION}

Currently great efforts go into the development of the initial value semiclassical (IVR) propagation techniques with applications ranging from the photodissociation and reactive scattering to condensed phase problems and nonadiabatic dynamics. Most methods are based on the propagator of Herman and Kluk (HK), ${ }^{1-3}$ related to the earlier "Frozen Gaussian" technique of Heller ${ }^{4}$ and "resurrected" through detailed theoretical studies by Kay. ${ }^{5-7}$ The first successful applications to the calculation of the $\mathrm{CO}_{2}$ photodissociation spectrum $^{8}$ and to dynamics of collinear $\mathrm{H}_{3}$ system ${ }^{9-11}$ drew attention to the great potential of the semiclassical propagation methods.

The semiclassical IVR methods for wavefunction time evolution are based on the propagation of classical trajectories that sample the phase space of an initial wave function without solving the initial/final value problem as in the Van Vleck-Gutzwiller propagator. ${ }^{12}$ The classical propagation is local, i.e., trajectories contribute to the final result (most often to a correlation function) independently of each other, which leads to negligible storage requirements. The semiclassical propagation can describe the quantum-mechanical effects with acceptable accuracy for a variety of problems. Now the HK propagator and its variations have been applied to reactive scattering, ${ }^{13}$ complex molecular systems, ${ }^{14-16}$ surface scattering, ${ }^{17}$ condensed phase, ${ }^{18-22}$ and nonadiabatic systems ${ }^{23-26}$ despite several undesirable features.

One of the drawbacks of the HK propagator is the dependence of the results on the initial parameters of a wave function and on the expansion parameter and the lack of an $a$ priori criterion for the reliability of semiclassical calculations. This problem is not getting attention it deserves. However, the fact that all studies with the HK propagator, with the exception of Refs. 27 and 28, attempt to merely reproduce quantum-mechanical results is evidence that further studies on the reliability of semiclassical methods are needed. The HK propagator (as other IVR propagators) is a phase space integral over initial conditions of classical trajectories evolving in time, that have classical actions as phases and functions of the stability matrix elements as amplitudes. These phases produce strong oscillations of the integrand and lead to slow convergence of the integral with respect to the number of trajectories. The problem of regularizing or " smoothing" the integrand was addressed in the number of works. The change of one component of the momentum for a time variable is used in Refs. 10, 29, and 30. Methods with additional approximations, such as the linearization, ${ }^{28,31}$ the stationary phase Monte Carlo integration, ${ }^{32}$ and the forward-backward propagation ${ }^{14,19}$ allow a reduction in the number of trajectories and help to obtain convergent results where straightforward application of the HK propagator is problematic. The convergence of the HK propagator was also discussed in Refs. 33-36.

In this paper we show that M $\phi$ ller operators can be conveniently combined with the HK propagator within the stationary phase approximation, which is inherent to the HK propagator. Mфller operators contain backward (forward) propagation in time under the asymptotic Hamiltonian and forward (backward) propagation under the full Hamiltonian, which yields substantial phase cancellation and reduction of the amplitude, as shown in Sec. II. This significantly improves the convergence properties of the HK propagator. In the numerical example (Sec. III) of the state-to-state reactive scattering for the collinear hydrogen exchange reaction, the number of trajectories is reduced by a factor of 10 compared to the calculation without Mфller operators. Section IV concludes. 


\section{MØLLER OPERATORS FOR SEMICLASSICAL REACTION PROBABILITY CALCULATIONS}

The HK propagator is an initial-value representation propagator that has the same semiclassical limit (i.e., the stationary phase approximation for $\hbar \rightarrow 0$ ) as the Schrödinger equation-the Van Vleck-Gutzwiller propagator. ${ }^{5,12}$ It is based on the expansion of a wavefunction in terms of Gaussians of a fixed width, whose centers move classically and whose phases are defined by the corresponding classical actions. The propagator is unitary in the stationary phase approximation, and it is time reversible. ${ }^{3}$ The HK propagator in $N$ dimensions, generalized to include the width parameters as a matrix, is

$$
\begin{aligned}
K^{\mathrm{sc}}\left(\mathbf{x}^{\prime}, t ; \mathbf{x}, 0\right)= & \frac{1}{(2 \pi)^{N}} \iint d \mathbf{p}_{0} d \mathbf{q}_{0} R_{p q t} e^{\imath S_{p q t}} \\
& \times g_{\gamma}\left(\mathbf{q}_{t}, \mathbf{p}_{t}, \mathbf{x}^{\prime}\right) g_{\gamma}^{*}\left(\mathbf{q}_{0}, \mathbf{p}_{0}, \mathbf{x}\right) .
\end{aligned}
$$

The function

$$
\begin{aligned}
g_{\gamma}\left(\mathbf{q}_{t}, \mathbf{p}_{t}, \mathbf{x}\right)= & \left(\frac{\operatorname{det}(\boldsymbol{\Gamma})}{\pi^{N}}\right)^{1 / 4} \\
& \times \exp \left(-\frac{1}{2}\left(\mathbf{x}-\mathbf{q}_{t}\right) \boldsymbol{\Gamma}\left(\mathbf{x}-\mathbf{q}_{t}\right)+\imath \mathbf{p}_{t} \cdot\left(\mathbf{x}-\mathbf{q}_{t}\right)\right),
\end{aligned}
$$

is a complex Gaussian with the diagonal width matrix $\boldsymbol{\Gamma}$ $=\left\{\gamma_{i}\right\}$. All $\gamma_{i}$ are positive real parameters. In theory, the propagator does not depend on the parameters $\left\{\gamma_{i}\right\}$. Vectors $\mathbf{q}_{0}=\left(q_{0}^{1}, \ldots, q_{0}^{N}\right)$ and $\mathbf{p}_{0}=\left(p_{0}^{1}, \ldots, p_{0}^{N}\right)$ are initial conditions of a classical trajectory at time zero and vectors $\mathbf{q}_{t}$ $=\left(q_{t}^{1}, \ldots, q_{t}^{N}\right)$ and $\mathbf{p}_{t}=\left(p_{t}^{1}, \ldots, p_{t}^{N}\right)$ are its coordinates and momenta at time $t$. $S_{p q t}$ is the classical action

$$
S_{p q t}=\int_{0}^{t}\left[\mathbf{p}_{t^{\prime}} \cdot \dot{\mathbf{q}}_{t^{\prime}}-H\left(\mathbf{p}_{t^{\prime}}, \mathbf{q}_{t^{\prime}}, t^{\prime}\right)\right] d t^{\prime} .
$$

The prefactor involving the stability (or monodromy) matrix elements is

$$
R_{p q t}=\sqrt{\operatorname{det}(\mathbf{B})},
$$

with the matrix elements $\mathbf{B}=\left\{b_{i j}\right\}$ being

$$
\begin{aligned}
b_{i j}= & \frac{1}{2}\left(\sqrt{\frac{\gamma_{i}}{\gamma_{j}}} \frac{\partial p_{t}^{i}}{\partial p_{0}^{j}}+\sqrt{\frac{\gamma_{j}}{\gamma_{i}}} \frac{\partial q_{t}^{i}}{\partial q_{0}^{j}}\right. \\
& \left.-\imath \sqrt{\gamma_{i} \gamma_{j}} \frac{\partial q_{t}^{i}}{\partial p_{0}^{j}}+\frac{\imath}{\sqrt{\gamma_{i} \gamma_{j}}} \frac{\partial p_{t}^{i}}{\partial q_{0}^{j}}\right) .
\end{aligned}
$$

The square root in Eq. (4) is chosen to make $R_{p q t}$ a continuous function of time. ${ }^{5}$ The oscillations of the integrand in Eq. (1), due to the classical action term and due to the complex prefactor $R_{p q t}$ whose amplitude grows with time, result in a large number of trajectories being needed to obtain convergence.

The wave packet correlation function approach ${ }^{37}$ with the IVR propagator, given by Eq. (1), is convenient and advantageous for the semiclassical calculation of reaction probabilities. Localized wave packets effectively make the limits of integration over $\mathbf{p}_{0}$ in Eq. (1) finite. The scattering matrix elements as a function of energy $E, S_{\beta \alpha}(E)$, can be obtained from the Fourier transform of the reactant/product wave packet correlation functions,

$$
C_{\alpha \beta}(t)=\left\langle\Phi_{\beta}^{-}\left|e^{-\imath H t}\right| \Phi_{\alpha}^{+}\right\rangle,
$$

as

$$
S_{\beta \alpha}(E)=\frac{(2 \pi)^{-1}}{\zeta_{\beta}^{*}(E) \eta_{\alpha}(E)} \int_{-\infty}^{\infty} C_{\alpha \beta}(t) e^{\imath E t} d t .
$$

The reactant wave packet $\left|\Phi_{\alpha}^{+}\right\rangle$is chosen so, that it evolves into a product of an incoming packet in the translational degree of freedom and an $\alpha$ eigenstate of the asymptotic internal Hamiltonian of reactants, when propagated into the infinite past, $t \rightarrow-\infty .{ }^{37}$ Similarly, the product wave packet $\left|\Phi_{\beta}^{-}\right\rangle$has to be separable into a product of an outgoing translational wave packet and a single internal eigenstate $\beta$ of the asymptotic internal Hamiltonian of products, when evolving into the infinite future, $t \rightarrow \infty$. Wave packets $\left|\Phi_{\alpha}^{+}\right\rangle$and $\left|\Phi_{\beta}^{-}\right\rangle$are constructed via Mфller operators,

$$
\Omega_{\alpha}^{+}=\lim _{t \rightarrow \infty} e^{-\imath H t} e^{\imath H_{\alpha}^{0} t} \quad \text { and } \Omega_{\beta}^{-}=\lim _{t \rightarrow \infty} e^{\imath H t} e^{-\imath H_{\beta}^{0} t},
$$

as

$$
\left|\Phi_{\alpha}^{+}\right\rangle=\Omega_{\alpha}^{+}\left|g^{+}(R) \times \chi_{\alpha}(r)\right\rangle
$$

and

$$
\left|\Phi_{\beta}^{-}\right\rangle=\Omega_{\beta}^{-}\left|g^{-}\left(R^{\prime}\right) \times \chi_{\beta}\left(r^{\prime}\right)\right\rangle .
$$

$H_{\alpha}^{0} / H_{\beta}^{0}$ are the asymptotic Hamiltonians for reactants/ products. Functions $g^{+}(R) / g^{-}\left(R^{\prime}\right)$ are incoming/outgoing localized wave packets in the translational coordinates in the appropriate asymptotic regions, and $\chi_{\alpha}(r) / \chi_{\beta}\left(r^{\prime}\right)$ are the eigenstates of the asymptotic internal Hamiltonians of reactants/products. The energy expansion coefficients $\eta_{\alpha}(E)$ and $\zeta_{\beta}(E)$ in Eq. (7) can be found analytically or numerically. ${ }^{37,38}$

To avoid confusion we take all times $t>0$ and use the appropriate sign in the propagators to determine "forward" and "backward." The operators $\Omega_{\alpha}^{+} / \Omega_{\beta}^{-}$propagate a wavefunction backward/forward in time under the asymptotic Hamiltonians $H_{\alpha}^{0} / H_{\beta}^{0}$ and then forward/backward in time under the full Hamiltonian $H$. This reduces the effect of the asymptotic channel propagation on the wave packets, i.e., the spreading and the phase accumulation.

In all prior applications, except Refs. 39 and 40, functions $g^{+}(R) / g^{-}\left(R^{\prime}\right)$ were originally chosen to have nonzero amplitude only in the reactant/product asymptotic regions of $H$,

$$
\left|\Phi_{\alpha}^{+}\right\rangle^{\mathrm{as}}=\left|g^{+}(R) \times \chi_{\alpha}(r)\right\rangle
$$

and

$$
\left|\Phi_{\beta}^{-}\right\rangle^{\text {as }}=\left|g^{-}\left(R^{\prime}\right) \times \chi_{\beta}\left(r^{\prime}\right)\right\rangle,
$$

so that Mфller operators had no effect. In general, if the original $g^{+}(R) / g^{-}\left(R^{\prime}\right)$ are localized where $H$ is different from the asymptotic Hamiltonians, the Møller operators will 
generate non-separable $\left|\Phi_{\alpha}^{+}\right\rangle$and $\left|\Phi_{\beta}^{-}\right\rangle$located in the interaction region of full $H$. The evolution operators in Eq. (6) with $\left|\Phi_{\alpha}^{+}\right\rangle$and $\left|\Phi_{\beta}^{-}\right\rangle$, given by Eq. (9), can be arranged as follows. $\Omega_{\alpha}^{+}$propagates $\left|g^{+} \times \chi_{\alpha}\right\rangle$ with $H_{\alpha}^{0}$ back in time from time zero to $t^{-}$, and then forward in time with the full $H$ until time zero. $\Omega_{\beta}^{-}$propagates $\left|g^{-} \times \chi_{\beta}\right\rangle$ forward in time under $H_{\beta}^{0}$ from time zero to $t^{+}$, and then back in time under full $H$ until time zero. The times $t^{-}$and $t^{+}$have to be sufficiently long, so, that with the purely incoming/outgoing $g^{+}(R) / g^{-}\left(R^{\prime}\right)$, the wavepackets $\left|e^{\imath H_{\alpha^{0}}^{0} t^{+}} g^{+} \times \chi_{\alpha}\right\rangle$ and $\left|e^{-\imath H_{\beta^{+}}^{0}} g^{-} \times \chi_{\beta}\right\rangle$ are located entirely in the respective asymptotic regions. The propagation under full $H$ of $\Omega_{\alpha}^{+}$and $\Omega_{\beta}^{-}$can be combined with the time propagation in Eq. (6) to give

$C_{\alpha \beta}(t)=\left\langle g^{-} \times \chi_{\beta}\left|e^{\imath H_{\beta^{-}}^{0}} e^{-\imath H\left(t^{-}+t+t^{+}\right)} e^{\imath H_{\alpha}^{0} t^{+}}\right| g^{+} \times \chi_{\alpha}\right\rangle$.

Equation (10) can be readily and efficiently combined with approximate propagators, based on classical trajectories, such as the IVR propagator of Herman and Kluk. The HK propagator, given by Eq. (1), is unitary within the stationary phase approximation, ${ }^{3}$

$$
K^{\mathrm{sc}}\left(t_{2}, t_{0}\right) \approx K^{\mathrm{sc}}\left(t_{2}, t_{1}\right) K^{\mathrm{sc}}\left(t_{1}, t_{0}\right) .
$$

Within this approximation, we can use a single set of classical trajectories for the total propagation in Eq. (10), including the propagation under $H_{\alpha}^{0}$ and $H_{\beta}^{0}$, because the transitions from $H_{\alpha}^{0}$ to $H$ and from $H$ to $H_{\beta}^{0}$ take place while all (reactive) trajectories are in the asymptotic regions, where $H$ is equivalent to $H_{\alpha}^{0}$ or to $H_{\beta}^{0}$. So, the classical trajectory, sampling $\left|g^{+} \times \chi_{\alpha}\right\rangle$, undergoes the following:

(1) It is propagated back in time under $H_{\alpha}^{0}$ until it reaches the asymptotic region of the reactants for time $t^{+}$;

(2) it is propagated forward under the full $H$ for time $T$ until it reaches the asymptotic region of products or reactants [here $T$ is the combined propagation time $t^{-}+t+t^{+}$of Eq. (10)];

(3) if the trajectory is reactive, it is propagated back in time under $H_{\beta}^{0}$ for time $t^{-}$until the trajectory stops contributing to the overlap with $\left\langle g^{-} \times \chi_{\beta}\right|$, as illustrated in Fig. 1.

The propagation times $t^{+}, T$, and $t^{-}$are specific for a trajectory, which contributes to the correlation function at time $t=T-t^{+}-t^{-}$. The procedure, though requiring longer total propagation per trajectory, will remove the effect of the asymptotic channel propagation on the trajectory contribution, thus, reducing the phase oscillations and decreasing the amplitude of $R_{p q t}$ which is dependent on the stability matrix elements. Smoothing the oscillations of the integrand by applying Møller operators improves the convergence of the results for semiclassical reactive scattering calculations.

\section{APPLICATION TO $\mathrm{H}_{2}+\mathrm{H}$ AND DISCUSSION}

We applied the Mbller operators to a collinear hydrogen exchange reaction. The details of the calculation are the same as in Ref. 10 except that the initial and final wave packets are
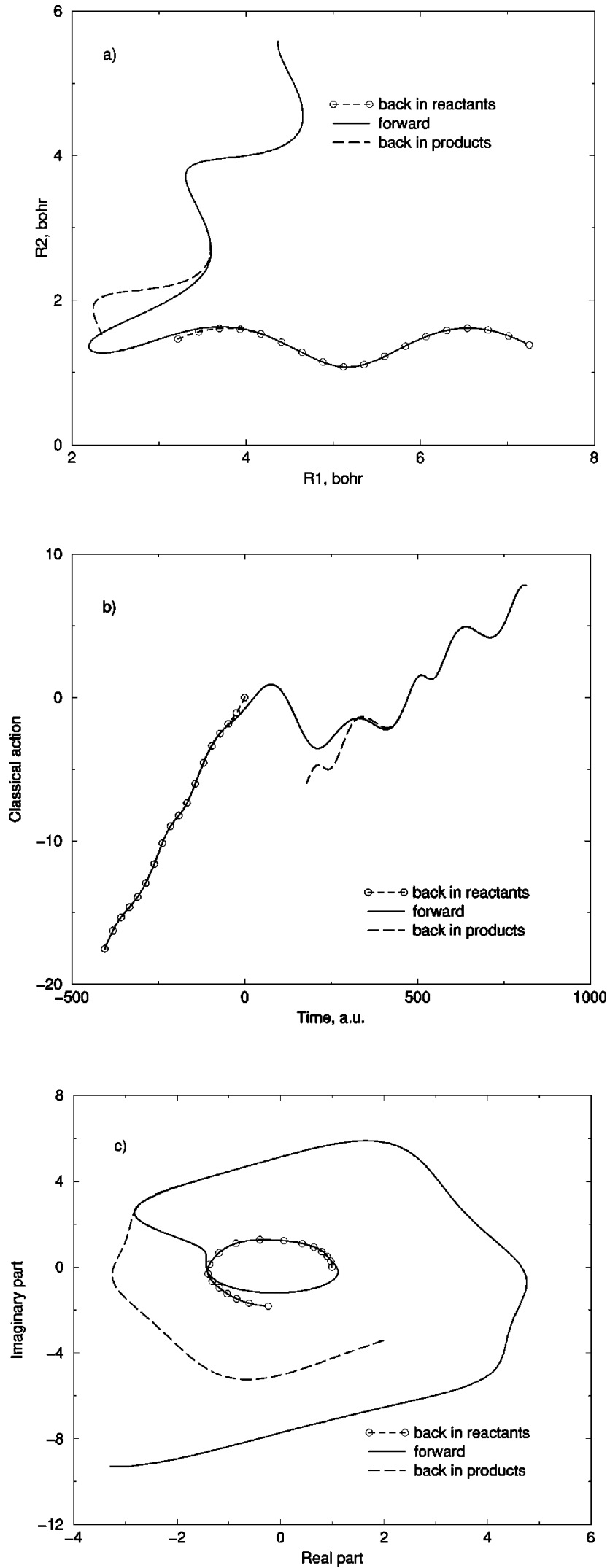

FIG. 1. Semiclassical application of Møller operators: (a) Classical trajectory propagated back in time with the asymptotic Hamiltonian for reactants, forward in time with the full Hamiltonian and back in time with the asymptotic Hamiltonian of products; (b) its classical action as a function of time; (c) the real and imaginary parts of its $R_{p q t}\left[R_{p q t}=(1,0)\right.$ at $\left.I^{+}=0\right]$. 


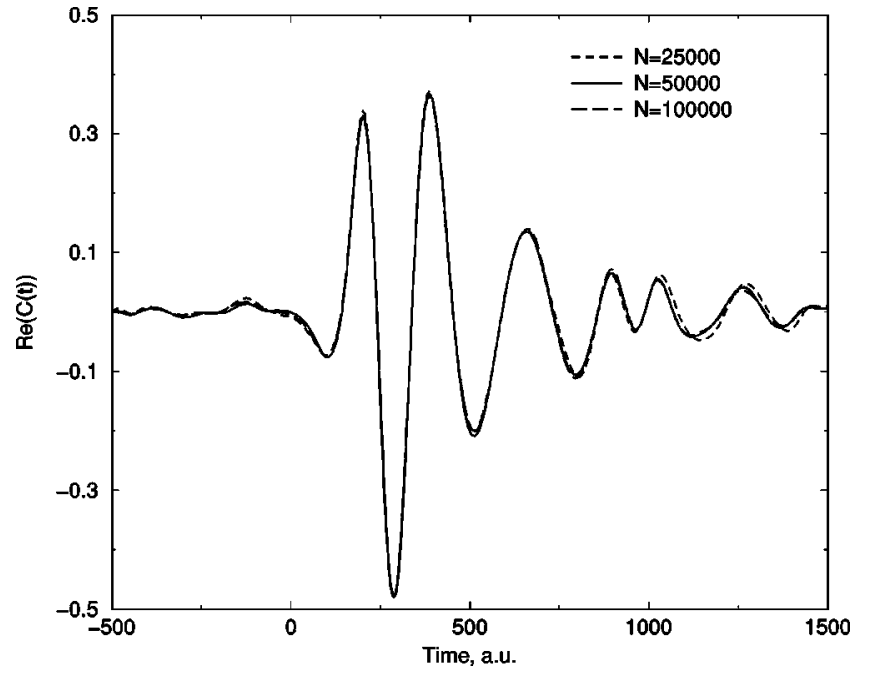

FIG. 2. Convergence of the correlation function with the number of trajectories: the real parts of the wave packet correlation functions obtained with $N=2.5 \times 10^{4}, 5 \times 10^{4}$, and $10^{5}$ trajectories.

centered at $R_{A(B)}=+(-) 3.0 \mathrm{bohr}$ in the translational degree of freedom, the width is $\gamma / 2=6 \mathrm{bohr}^{-2}$ and the initial (final) momentum in translation is $p_{A(B)}=-(+) 7.0 . S_{00}$ only is presented here. Figure 1 shows a representative reactive trajectory and its phase $S_{p q t}$ and the prefactor $R_{p q t}$. With the Møller operators we obtained converged results with 5 $\times 10^{4}$ classical trajectories which is 10 times fewer trajectories than was required with the initial and final wave packets defined in the asymptotic regions of a potential surface in Ref. 10. Figure 2 shows the real parts of the correlation functions for the $0 \rightarrow 0$ transition obtained with $25 \times 10^{3}, 50$ $\times 10^{3}$, and $100 \times 10^{3}$ trajectories. Results for two larger calculations are hardly distinguishable on the plot. Figure 3 shows quantum-mechanical semiclassical probabilities of the $0 \rightarrow 0$ reactive transition.

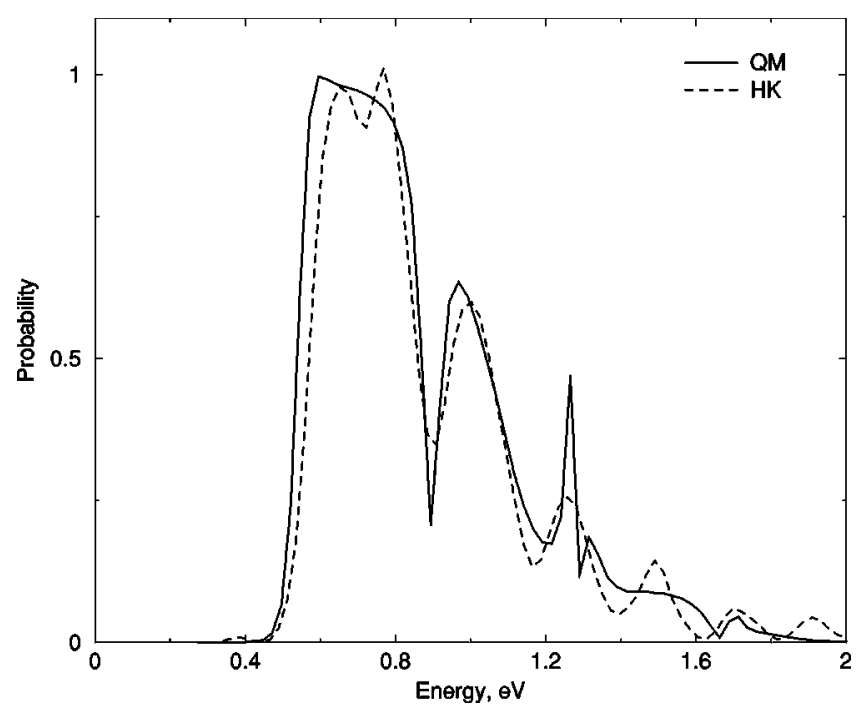

FIG. 3. Reaction probability for the $0 \rightarrow 0$ transition for the collinear hydrogen exchange reaction obtained from the quantum-mechanical (QM) correlation function and semiclassical (HK) correlation function with Mbller operators.

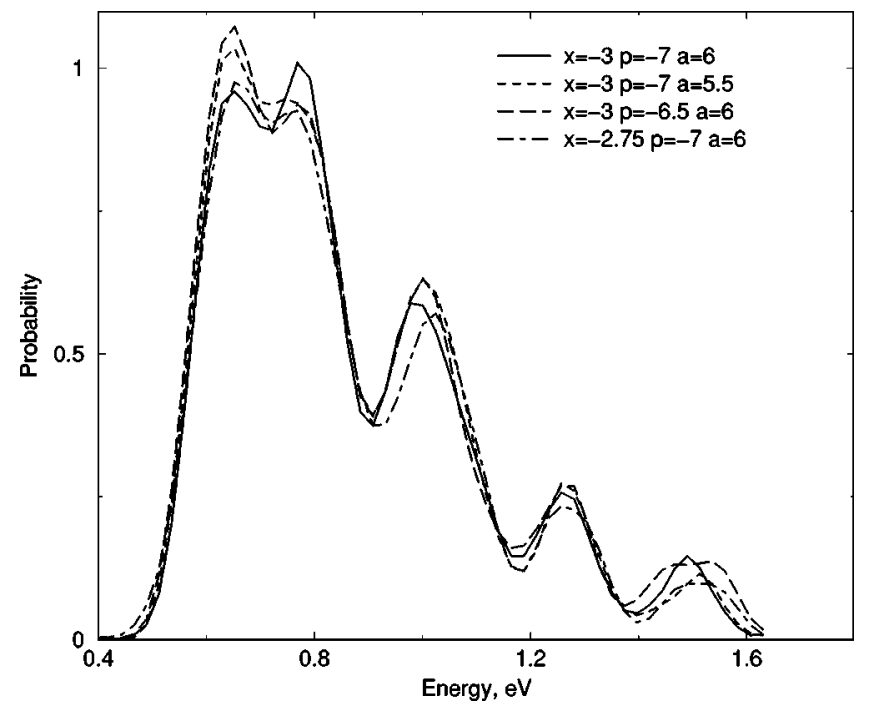

FIG. 4. Dependence of the semiclassical probability for the $0 \rightarrow 0$ transition on the parameters of the initial wave packet. On the legend $x$ is the position, $p$ is the momentum, and $a$ is the width parameter of the initial translational wave packet.

We also note that though our semiclassical results are fully converged with respect to the number of trajectories, the semiclassical reaction probabilities do depend on the parameters of the initial and final wave packets defined with or without Møller operators. The criterion for choosing the wave packet parameters is the best cancellation of the phases in the propagator: the wave packets are defined in the interaction region of the full $H$, but not far into the region where actual bond-breaking occurs. The wave packet has to be compact in translation to cover a wide range of energies and not to go far into the interaction and asymptotic regions. The initial translational momentum is chosen so that the wave packet is incoming and covers the desirable range of energies. Figure 4 illustrates the dependence of the transition probability on the wave packet parameters.

The outlined strategy of using Møller operators can be readily extended to the calculation of the cumulative reaction probability in terms of the Fourier transforms of the wave packet correlation functions ${ }^{38}$ and to other IVR propagators. It is expected to be particularly useful for systems with long range interactions.

\section{SUMMARY}

In this work we suggest and use the Mфller operators in conjunction with the semiclassical HK propagator without making further approximations, beyond the ones inherent to the semiclassical propagator, which improves the convergence properties of the semiclassical method. Møller operators, where applicable, reduce oscillations and the amplitude of the phase space integrals in IVR propagators. Thus, the number of classical trajectories was decreased tenfold compared to the calculation without Møller operators in the test calculation of the state-to-state reaction probabilities for the collinear $\mathrm{H}_{3}$ system. Convergence with respect to the number of trajectories was achieved. However, semiclassical probabilities show some dependence on the parameters of calcu- 
lation, such as the particular form of the initial and final wave packets. This question needs further studies if semiclassical methods are to become a reliable computational tool for detailed quantum properties, such as state-to-state reaction probabilities, in the future.

\section{ACKNOWLEDGMENT}

This research was supported in part by a grant from the Department of Energy, DE-FG02-87ER13679.

${ }^{1}$ M. F. Herman and E. Kluk, Chem. Phys. 91, 27 (1984).

${ }^{2}$ E. Kluk, M. F. Herman, and H. L. Davis, J. Chem. Phys. 84, 326 (1986).

${ }^{3}$ M. F. Herman, J. Chem. Phys. 85, 2069 (1986).

${ }^{4}$ E. Heller, J. Chem. Phys. 75, 2923 (1981).

${ }^{5}$ K. G. Kay, J. Chem. Phys. 100, 4377 (1994).

${ }^{6}$ K. G. Kay, J. Chem. Phys. 100, 4432 (1994).

${ }^{7}$ K. G. Kay, J. Chem. Phys. 101, 2250 (1994).

${ }^{8}$ A. Walton and D. E. Manolopoulos, Chem. Phys. Lett. 244, 448 (1995).

${ }^{9}$ F. Grossmann, Chem. Phys. Lett. 262, 470 (1996).

${ }^{10}$ S. Garashchuk and D. J. Tannor, Chem. Phys. Lett. 262, 477 (1996).

${ }^{11}$ S. Garashchuk, F. Grossmann, and D. J. Tannor, J. Chem. Soc., Faraday Trans. 93, 781 (1997).

${ }^{12}$ M. Gutzwiller, Chaos in Classical and Quantum Mechanics (SpringerVerlag, New York, 1990).

${ }^{13}$ D. E. Skinner and W. H. Miller, Chem. Phys. Lett. 399, 20 (1999).

${ }^{14}$ X. Sun and W. H. Miller, J. Chem. Phys. 110, 6635 (1999).
${ }^{15}$ W. H. Miller, J. Chem. Phys. 103, 9384 (1999).

${ }^{16}$ T. B. Wang, M. Thoss, and W. H. Miller, J. Chem. Phys. 112, 47 (2000).

${ }^{17}$ D. A. McCormack, J. Chem. Phys. 112, 992 (2000).

${ }^{18}$ N. Makri and K. Thompson, Chem. Phys. Lett. 291, 101 (1998).

${ }^{19}$ K. Thomson and N. Makri, J. Chem. Phys. 110, 1343 (1999).

${ }^{20}$ J. S. Shao and N. Makri, J. Phys. Chem. A 103, 7753 (1999).

${ }^{21}$ J. S. Shao and N. Makri, J. Phys. Chem. A 103, 9479 (1999).

${ }^{22}$ O. Kuhn and N. Makri, J. Phys. Chem. A 103, 9487 (1999).

${ }^{23}$ X. Sun and W. H. Miller, J. Chem. Phys. 108, 8870 (1998).

${ }^{24}$ X. Sun and W. H. Miller, J. Chem. Phys. 106, 6346 (1997).

${ }^{25}$ E. A. Coronado, V. S. Batista, and W. H. Miller, J. Chem. Phys. 112, 5566 (2000).

${ }^{26}$ F. Grossmann, Phys. Rev. A 60, 1791 (1999).

${ }^{27}$ V. A. Apkarian and N. Schwentner, Chem. Rev. 99, 1481 (1999).

${ }^{28}$ A. Walton and D. E. Manolopoulos, Mol. Phys. 87, 961 (1996).

${ }^{29}$ Y. Elran and K. G. Kay, J. Chem. Phys. 110, 3653 (1999).

${ }^{30}$ Y. Elran and K. G. Kay, J. Chem. Phys. 110, 8912 (1999).

${ }^{31}$ M. L. Brewer, J. S. Hulme, and D. E. Manolopoulos, J. Chem. Phys. 106, 4832 (1997).

${ }^{32}$ M. F. Herman, Chem. Phys. Lett. 275, 445 (1998).

${ }^{33}$ B. E. Guerin and M. F. Herman, Chem. Phys. Lett. 286, 361 (1998).

${ }^{34}$ M. F. Herman and D. F. Coker, J. Chem. Phys. 111, 1801 (1999).

${ }^{35}$ B. R. McQuarrie and P. Brumer, Chem. Phys. Lett. 319, 27 (2000).

${ }^{36}$ M. L. Brewer, J. Chem. Phys. 111, 6168 (1999).

${ }^{37}$ D. J. Tannor and D. E. Weeks, J. Chem. Phys. 98, 3884 (1993).

${ }^{38}$ S. Garashchuk and D. J. Tannor, J. Chem. Phys. 110, 2761 (1999).

${ }^{39}$ D. E. Weeks and D. J. Tannor, Chem. Phys. Lett. 207, 301 (1993).

${ }^{40}$ D. E. Weeks and D. J. Tannor, Chem. Phys. Lett. 224, 451 (1994). 